\title{
Conductive and convective heat transfer in fluid flows between differentially heated and rotating cylinders
}

\author{
Jose M. Lopez ${ }^{\mathrm{a}, \mathrm{b}, *}$, Francisco Marques ${ }^{\mathrm{a}}$, Marc Avila ${ }^{\mathrm{b}}$ \\ ${ }^{a}$ Department of Física Aplicada, Universitat Politècnica de Catalunya, Girona s/n, Mòdul B4 Campus Nord, 08034 \\ Barcelona, Spain \\ ${ }^{b}$ Institute of Fluid Mechanics, Department of Chemical and Biological Engineering, Friedrich-Alexander-Universität \\ Erlangen-Nürnberg, 91058 Erlangen, Germany
}

\begin{abstract}
The flow of fluid confined between a heated rotating cylinder and a cooled stationary cylinder is a canonical experiment for the study of heat transfer in engineering. The theoretical treatment of this system is greatly simplified if the cylinders are assumed to be of infinite length or periodic in the axial direction, in which cases heat transfer occurs only through conduction as in a solid. We here investigate numerically heat transfer and the onset of turbulence in such flows by using both periodic and no-slip boundary conditions in the axial direction. The influence of the geometric parameters is comprehensively studied by varying the radius ratio $(0: 1 \leq \eta \leq 0: 99)$ and the length-to-gap aspect ratio $(5 \leq \Gamma \leq 80)$. Similarly, a wide range of Prandtl, Rayleigh, and Reynolds numbers is explored $(0: 0 \leq \sigma \leq 100 ; R a \leq 30000$, and $R e \leq 1000$, respectively). We obtain a simple linear criterion, $R a \lesssim a(\eta) \Gamma$, which determines whether the infinite-cylinder assumption can be employed. The coefficient $a$ is well approximated by a cubic fit over the whole $\eta$-range. Noteworthy the criterion is independent of the Prandtl number and appears robust with respect to Reynolds number even beyond the laminar regime.
\end{abstract}

Keywords: Taylor-Couette, radial heating, end walls, heat transfer

\section{Introduction}

Instabilities driven by the combination of rotation and thermal gradients determine the dynamics of complex geophysical, astrophysical and industrial flows. Simple models of such flows can be tested in laboratory experiments of a laterally heated differentially rotating annulus, for which extensive information about the physical mechanisms and flow regimes can be found in the literature (see 1 for a review on this topic). The case of rotating heated inner cylinder and stationary cooled outer cylinder (RHISCO) is a model for the cooling of rotating machinery, the solidification of pure metals, techniques of chemical vapor deposition, rotating-tube heat exchangers and nuclear reactor fuel rods [2, 3, 4, 5]. The geometry of such an experimental apparatus is fully specified by the length-to-gap aspect ratio $\Gamma=h /\left(r_{o}-r_{i}\right)$, and the radius-ratio $\eta=r_{i} / r_{o}$, where $r_{i}$ and $r_{o}$ are the radii of the inner and outer cylinders, and $h$ is their height.

Depending on the geometry, RHISCO experiments used in the literature can be classified in two groups. The first group of experiments [6, 7, 8, is characterized by long cylinders $\Gamma=h /\left(r_{o}-r_{i}\right) \geq 100$ and narrow gap $\eta=r_{i} / r_{o} \lesssim 1$. Ali and Weidman [9] performed a detailed linear stability analysis of such flows using axial periodicity and reported on the influence of the Prandtl number $(\sigma)$ and $\eta$ on the stability boundaries. Their results showed a good agreement with [6] and, to a lesser extent with [7]. Ali and Weidman attributed the discrepancies to the limitations of linear stability theory and the infinite-cylinder idealisation to capture the experimental details. A similar linear stability analysis [10] reported good agreement between numerical

\footnotetext{
* Corresponding author

Email address: jmlopez@fa.upc.edu (Jose M. Lopez)
} 
and related experimental results $[\underline{8}$. Nonlinear simulations for small temperature gradients were provided by Kedia et al. [1] who quantified the heat transfer across the system. A second group of experiments embraces apparatuses with moderate aspect ratio and wide gap. Ball and Farouk 12, 13, 14 reported heat transfer measurements as well as the sequence of flow transitions using an experimental setup with $\Gamma=31.5$ and $\eta \sim 0.5$. Subsequent numerical simulations 15 for $\Gamma=10$ and $\eta=0.5$ provided insight on the bifurcations structure of the system. However, the results showed significant discrepancies with experiments suggesting strong effects of the axial boundaries.

An accurate numerical simulation of the axial (Ekman) boundary layers in flows between long cylinders entails a substantial computational cost, especially for rapid rotation and large temperature gradients. The assumption of axial periodicity reduces the computational effort because the Ekman layers are not present and only a short central fraction of the apparatus needs to be simulated to estimate transport properties. Moreover, under this assumption variables can be expanded as a Fourier series in the axial direction, which greatly simplifies the numerical approach and enables the use of more efficient solvers.

In this paper we determine under what conditions periodic boundary conditions can be employed to describe the dynamics of RHISCO experiments. In particular, we provide criteria to distinguish flow features that arise from the interplay between differential rotation and temperature, from those which are mainly determined by the axial boundaries or end walls. We compare the flow dynamics by using both physical (noslip) and axially periodic boundary conditions in our numerical simulations. We show that axial periodicity renders a good approximation of laboratory flows as long as the Rayleigh number $R a$ is small. We provide a simple criterion that determines whether heat transfer in the laminar flow is conductive or convective. In particular, conductive profiles, which enable the use of axially periodic boundary conditions, are realised as long as the Rayleigh number satisfies $R a<a(\eta) \Gamma+b(\eta)$.

\section{Specification of the system and numerical methods}

We consider the motion of an incompressible fluid of kinematic viscosity $\nu$ and thermal diffusivity $\kappa$ confined in the annular gap between two rigid and concentric rotating cylinders of radii $r_{i}$ and $r_{o}$. The inner cylinder rotates at constant angular velocity $\Omega$, whereas the outer cylinder is kept at rest. A radial thermal gradient is considered by setting the inner and outer cylinder temperature to $T_{i}=\bar{T}+\Delta T / 2$ and $T_{o}=\bar{T}-\Delta T / 2$, respectively. Here $\bar{T}$ is the mean temperature of the fluid. The axis of the cylinders is vertical, i. e. parallel to the gravitational acceleration $g$. We study flows with stationary end walls and with axially periodic boundary conditions (Fig. 1. (a) and (b) respectively). The latter model the case of infinitely long cylinders, whereas the former reproduce experimental boundary conditions (no-slip for the velocity and thermally insulating end walls for the temperature).

\subsection{Governing equations}

We consider the Boussinesq approximation including centrifugal buoyancy in an inertial reference frame as described in [16. The dimensionless governing equations are

$$
\begin{aligned}
& \left(\partial_{t}+\mathbf{v} \cdot \nabla\right) \mathbf{v}=-\nabla p+\nabla^{2} \mathbf{v}+G T \hat{\mathbf{z}}+\epsilon T \mathbf{v} \cdot \nabla \mathbf{v}, \\
& \left(\partial_{t}+\mathbf{v} \cdot \nabla\right) T=\sigma^{-1} \nabla^{2} T \\
& \nabla \cdot \mathbf{v}=0
\end{aligned}
$$

where $\mathbf{v}=(u, v, w)$ denotes the velocity field vector and $T$ is the deviation of the temperature with respect to $\bar{T}$. The length, time, temperature and pressure scales chosen to make the set of equations dimensionless are the gap width $d=r_{o}-r_{i}$, the viscous time $d^{2} / \nu$, the temperature difference between the cylinders $\Delta T$ and $(\nu / d)^{2}$ respectively. There are six independent dimensionless numbers (see table 1). The term $\epsilon T \mathbf{v} \cdot \nabla \mathbf{v}$ accounts for centrifugal buoyancy, including secondary effects stemming from differential rotation or strong internal vorticity [16]. The equations are solved in cylindrical coordinates $(r, \theta, z)$ 
$(a)$

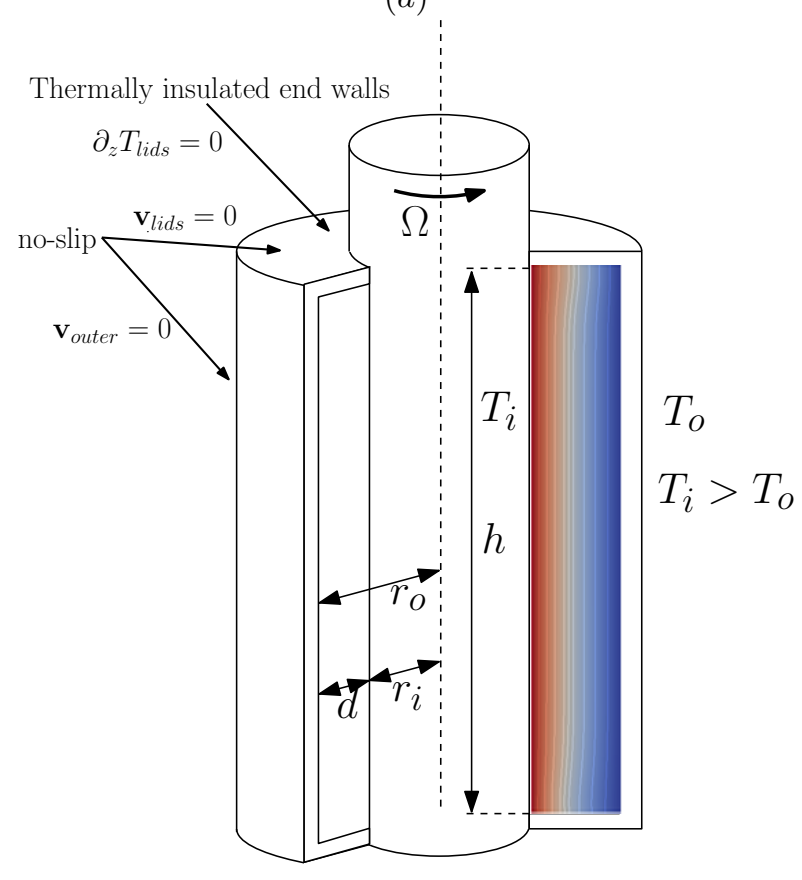

(b)

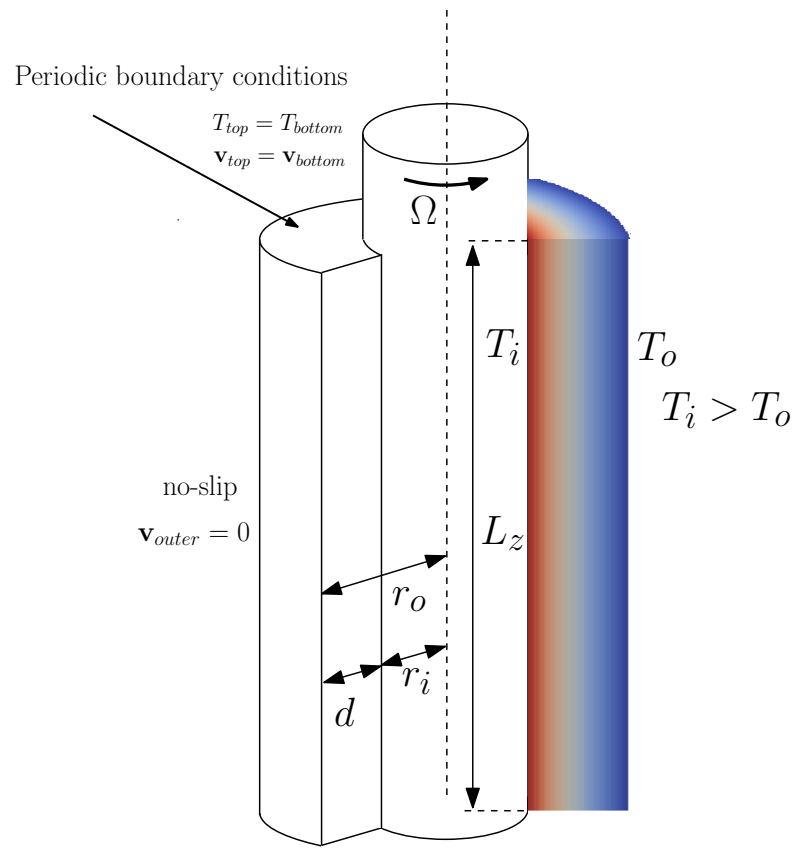

Figure 1: Sketches of geometry and boundary conditions for the two cases considered in this paper. (a) Stationary insulating endwalls (no-slip boundary condition for the velocity and zero flux for the temperature) and (b) axially periodic boundary conditions. The temperature profile is superimposed on the right hand side of each figure.

\begin{tabular}{|c|c|c|c|}
\hline Name & Symbol & Formula & Operation range \\
\hline Grashof number & $G$ & $\alpha g \Delta T d^{3} / \nu^{2}$ & $0 \leq G \leq 12000$ \\
\hline Relative density variation & $\epsilon$ & $\alpha \Delta T=\Delta \rho / \rho_{0}$ & $0 \leq \epsilon \leq 0.025$ \\
\hline Prandtl number & $\sigma$ & $\nu / \kappa$ & $0.01 \leq \sigma \leq 100$ \\
\hline Aspect ratio & $\Gamma$ & $h / d$ & $5 \leq \Gamma \leq 80$ \\
\hline Radius ratio & $\eta$ & $r_{i} / r_{o}$ & $0.1 \leq \eta \leq 0.99$ \\
\hline Reynolds number & $R e$ & $\Omega r_{i} d / \nu$ & $0 \leq R e \leq 1000$ \\
\hline
\end{tabular}

Table 1: Dimensionless parameters. Here $\kappa$ and $\alpha$ are respectively the thermal diffusivity and the coefficient of volume expansion of the fluid, $\Delta \rho$ is the density variation associated with a temperature change of $\Delta T$. Last column indicates the range of values covered by the simulations shown in this paper. 


\subsection{Numerical methods}

In the axially periodic case the onset of instabilities was determined via linear stability analysis of the basic flow as in [16]. Fully nonlinear simulations were performed using the Boussinesq-approximation [16], which was added with the heat equation to the finite-difference-Fourier-Galerkin (hybrid MPI-OpenMP) code of Shi et al.[17. A time-step $\delta t=2 \times 10^{-5}$ viscous time units was used in all computations.

For rigid end walls the governing equations were solved using a second-order time-splitting method. A pseudo-spectral formulation is used for the spatial discretisation, with the Fourier-Galerkin method in the azimuthal coordinate $\theta$ and Chebyshev collocation in $r$ and $z$. The code is based on a previous hydrodynamic code [18, which has been extended with the Boussinesq-approximation of [16] and parallelised as in [17. Details can be found in J. M. Lopez's PhD thesis [19. The numerical resolution has been chosen to ensure that the infinite norm of the spectral coefficients decays at least four orders in magnitude. Time steps as small as $\delta t=1 \times 10^{-5}$ viscous time units have been required for numerical stability and accuracy of the second-order temporal scheme.

\section{Conductive and convective basic flows}

The assumption of axial periodicity allows to considerably simplify the calculation of the basic flow. The radial velocity is zero and the rest of variables only depend on the radial component. Under these conditions an analytical solution can be found by imposing a zero axial mass flux. This reads

$$
\begin{aligned}
& v_{b}(r)=A r+\frac{B}{r}, \\
& w_{b}(r)=G\left(C\left(r^{2}-r_{i}^{2}\right)+\left(C\left(r_{o}^{2}-r_{i}^{2}\right)+\frac{1}{4}\left(r_{o}^{2}-r^{2}\right)\right) \frac{\ln \left(r / r_{i}\right)}{\ln \eta}\right), \\
& T_{b}(r)=\frac{\ln \left(r / r_{o}\right)}{\ln \eta}-\frac{1}{2},
\end{aligned}
$$

The azimuthal velocity $v_{b}$ is the classical Couette flow, $T_{b}$ corresponds to the temperature in a conductive regime and $w_{b}$ is the axial velocity profile induced by gravitational buoyancy. The parameters $A, B$ and $C$ are

$$
\begin{aligned}
& A=\frac{R e_{o}-\eta R e}{1+\eta}, \quad B=\eta \frac{R e-\eta R e_{o}}{(1-\eta)\left(1-\eta^{2}\right)}, \\
& C=-\frac{4 \ln \eta+\left(1-\eta^{2}\right)\left(3-\eta^{2}\right)}{16\left(1-\eta^{2}\right)\left(\left(1+\eta^{2}\right) \ln \eta+1-\eta^{2}\right)},
\end{aligned}
$$

and the non-dimensional radii are $r_{i}=\eta /(1-\eta), r_{o}=1 /(1-\eta)$.

Note that the conductive temperature (2c) only depends on the radial geometry. Figure 2 illustrates the variation of the axial velocity and temperature with $\eta$.

\subsection{End walls effect: influence of fluid properties and geometry.}

The presence of end walls in the system leads to non-zero radial velocities close to the end walls, commonly referred to as Ekman layers, and enables convective heat transfer across the gap even for the basic flow [20, 21. This switches on the contribution of terms of the heat equation, which were zero under the axial periodicity assumption. The temperature equation $1 \mathrm{~b}$ reads

$$
\partial_{t} T_{b}+u_{b} \partial_{r} T_{b}+\frac{v_{b}}{r} \partial_{\theta} T_{b}+w_{b} \partial_{z} T_{b}=\sigma^{-1} \nabla^{2} T_{b},
$$

In the infinite case the steady basic flow has no radial velocity $\left(u_{b}=0\right)$ and the temperature $\left(T_{b}\right)$ depends only on $r$, so all terms in the left hand side are zero. Consequently, the basic flow is unaffected by changes in the fluid properties. In contrast, the influence of $\sigma$ may be expected to play a significant role in bounded 
(a)

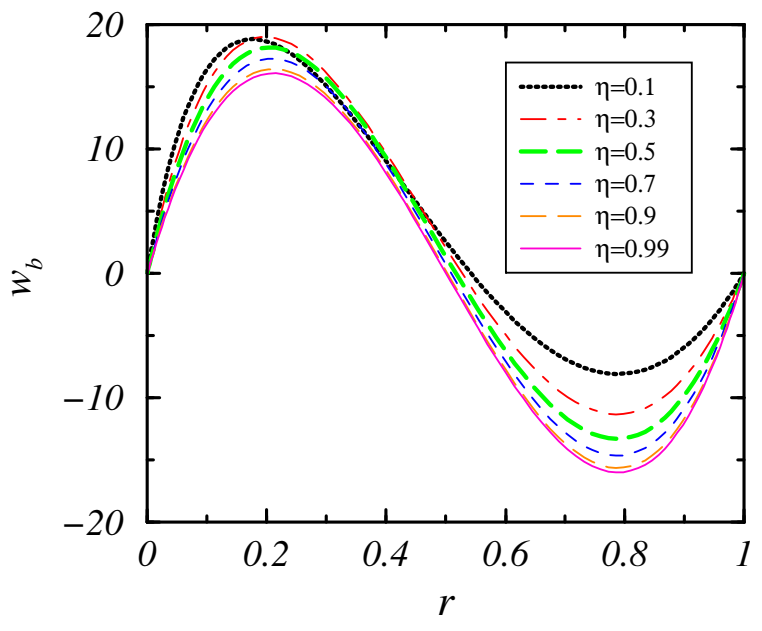

(b)

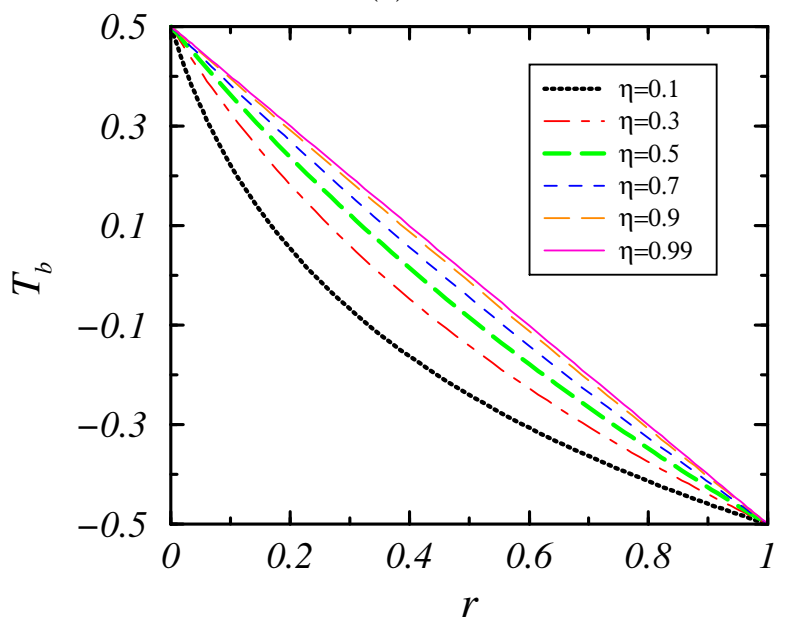

Figure 2: Radial profiles of $(a)$ axial velocity (computed for $G=2000)$ and $(b)$ temperature as a function of the radius ratio $\eta$.

systems. It modifies the basic flow with respect to the idealised periodic situation and may become an important source of discrepancies between both systems.

Figure 3 $(a)$ shows colormaps of the temperature in a meridional section $(r, z)$ for $\eta=0.5, R e=30$, $G=2000, \Gamma=10$, and $\sigma=0.1,1,10$. It clearly illustrates how changes in $\sigma$ alter the temperature of the basic flow in a finite system. Radial and axial temperature profiles are shown respectively in figures $(b)$ at mid-height and $(c)$ at mid-gap as a function of $\sigma$. For $\sigma \leq 1$ (gases or liquid metals), and sufficiently far from the end walls, the temperature profiles are nearly independent of $z$ and thus deviations from the conductive regime $(\sigma=0)$ are negligible. The axial dependence becomes stronger with increasing $\sigma$, resulting in radial temperature profiles that differ substantially from a conductive profile even at mid-height. This temperature regime is usually referred to as convective [22, 23, and is characterised by sharp thermal boundary layers and enhanced heat transfer.

The heat transfer across the gap is here characterised by the Nusselt of the inner cylinder

$$
<N u_{i}>_{t}=\frac{\left.\int_{0}^{2 \pi} \int_{-\Gamma / 2}^{\Gamma / 2} \partial_{r} T\right|_{r_{i}} \mathrm{~d} z \mathrm{~d} \theta}{q_{\text {cond }}}
$$

where $\left\langle.{ }_{t}\right.$ indicates time average and $q_{\mathrm{qcond}}$ denotes the dimensionless heat flux of the laminar (conductive) flow in the periodic case. To simplify the notation the subindex $i$ is omitted henceforth. Note that the Nusselt number of the outer cylinder is $N u_{o}=N u_{i} \eta$. The effect of the transition between the conductive and convective regime on heat transfer is shown in figure $3(d)$. Large $\sigma$ and short $\Gamma$ promote convective profiles and thus efficient heat transfer.

\subsection{Transition from conductive to convective: the Rayleigh effect}

We quantify the departure of the radial temperature profiles from the conductive regime with the parameter

$$
D=\frac{\int_{r_{i}}^{r_{o}}\left(T_{\text {mid }}-T_{\text {cond }}\right)^{2}}{\int_{r_{i}}^{r_{o}} T_{\text {cond }}^{2}}
$$

where $T_{\text {mid }}$ is the radial temperature profile at mid-height and $T_{\text {cond }}$ denotes the conductive profile (2c) of the infinite-cylinder case. 

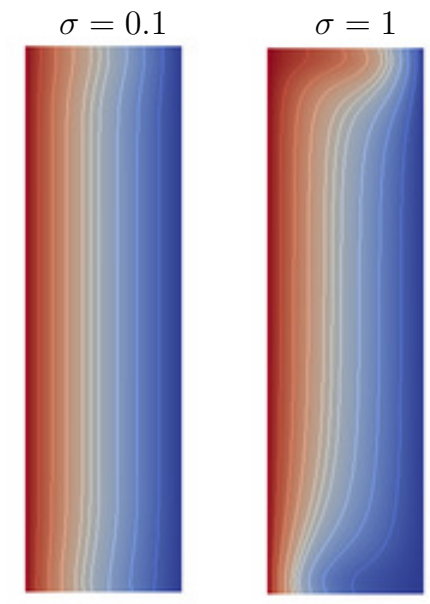

(a)

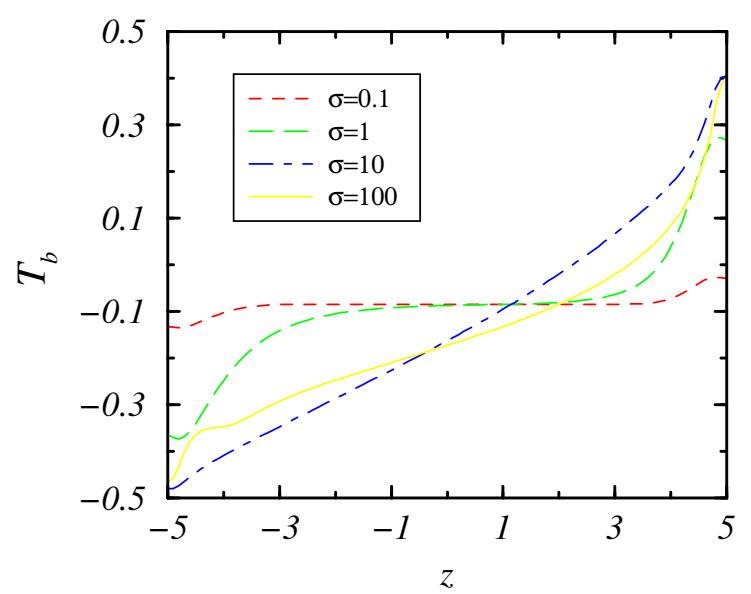

(c)

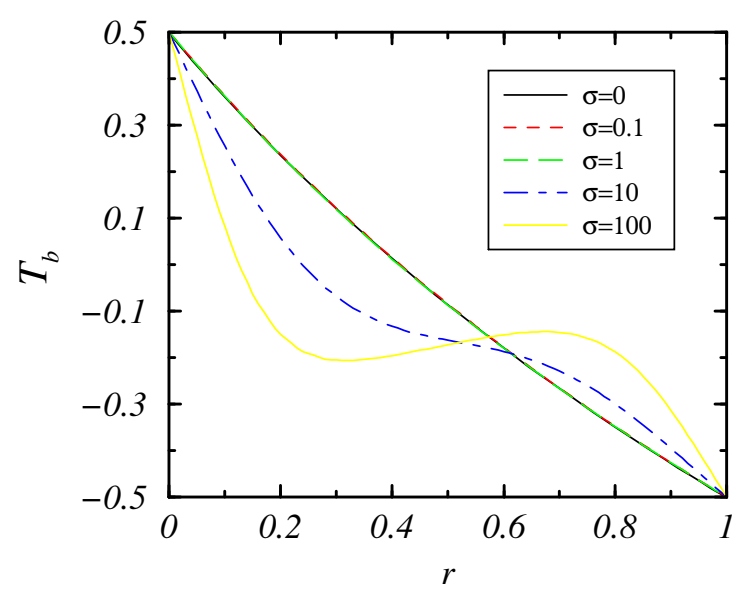

(b)

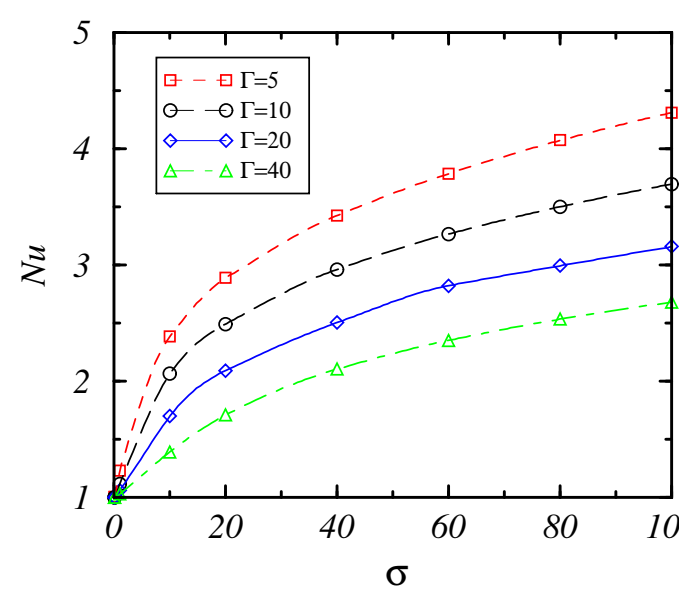

(d)

Figure 3: (color online) (a) Colormaps of the temperature for $\eta=0.5, R e=30, G=2000, \Gamma=10$ and different values of $\sigma$. They are plotted in a meridional section $(r, z)$, being the inner cylinder on the left hand side. Note that the aspect-ratio has been changed in these plots to facilitate the visualization of the contours. Temperature profiles for different values of $\sigma$ at mid-height $(b)$ and mid-gap $(c)$ (other parameters remain as in $(b)$ ). The case of $\sigma=0$ corresponds to the axially periodic system. The variation of $N u$ with $\sigma$ for several values of $\Gamma$ (other parameters remain equal) are shown in $(d)$. 
(a)

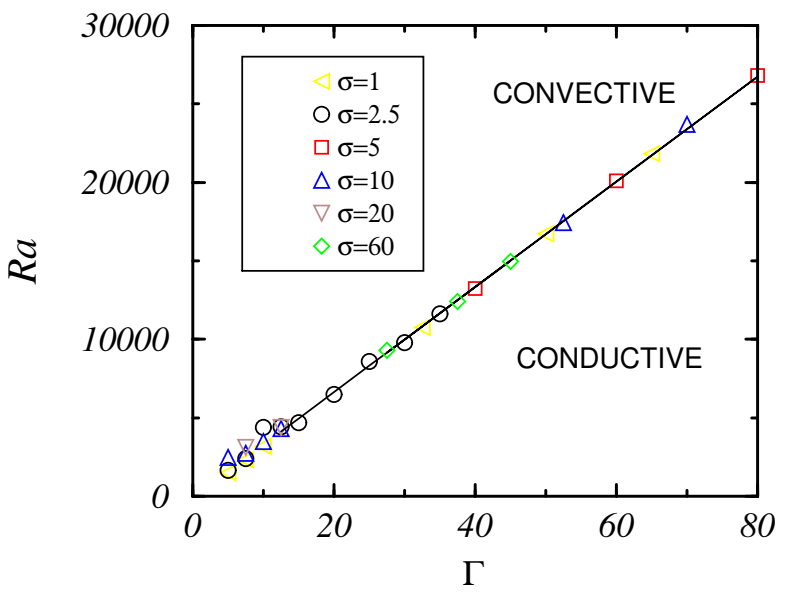

(b)

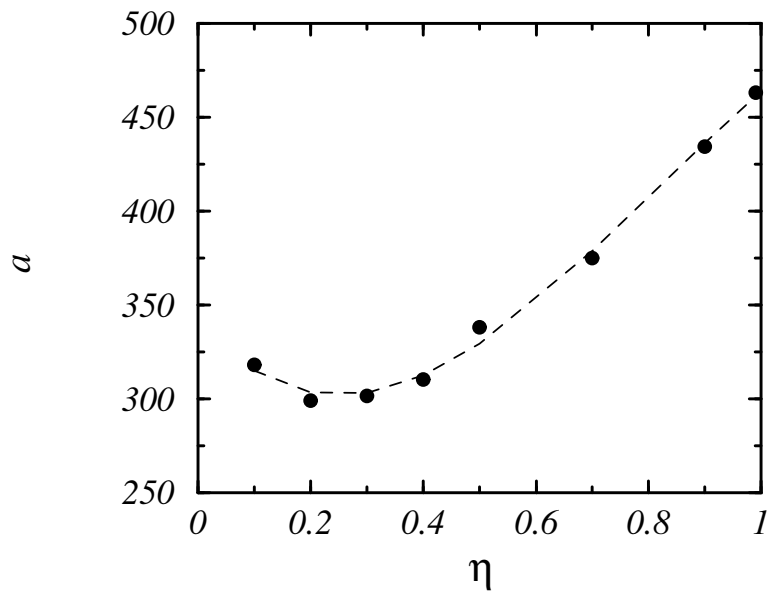

Figure 4: (color online) (a) Transition from conductive to convective temperature regime $\left(D \gtrsim 10^{-3}\right)$ as a function of the aspect-ratio $\Gamma$ and Rayleigh number $R a$ for $\eta=0.5$. (b) Variation of the slope $a$ of the linear equation describing the transition between the conductive and convective regimes with $\eta$.

Figure 4 (a) shows the transition between the conductive and convective regimes as a function of the Rayleigh number $(R a=\sigma G)$ and $\Gamma$. The critical Rayleigh numbers $R a_{c}$ have been computed by fixing $\sigma$ (using different values) and $\Gamma$, and increasing $G$ until $D \gtrsim 10^{-3}$ was reached. For low values of $\Gamma$ there are significant differences between $R a_{c}$ calculated with different $\sigma$. However, for $\Gamma \gtrsim 12.5$ the transition takes place at nearly the same $R a_{c}$ regardless of $\sigma$. This interesting behaviour can be explained by analysing equation (2c). The laminar state is steady and axisymmetric, so departures from the conductive regime are due to either $u_{b} \partial_{r} T_{b}$ or $w_{b} \partial_{z} T_{b}$. The first term is only affected by changes in $\sigma$ and plays a significant role in setups with a relatively short aspect-ratio, as the radial velocities arising near the end walls are more vigorous. Consequently, variations of $R a_{c}$ with $\sigma$ are observed in short setups. In contrast, the second term depends on both $\sigma$ and $G$, but only through their product: the Rayleigh number $R a$. This becomes progressively dominant as $\Gamma$ increases and the transition to the conductive regime becomes dependent on $R a$ only. Interestingly, $R a_{c}$ varies linearly with $\Gamma$ according to

$$
R a_{c}(\Gamma)=338.04 \Gamma-216.24
$$

This linear behaviour was also observed for fixed $\sigma=1$ by Thomas and De Vhal Davis 22, who studied the transition between both temperature regimes for natural convection.

The linear relationship (8) separates the regions of parameter space in which the temperature regime is conductive and conductive. It provides a criterion to determine a priori whether simulations using axially periodic boundary conditions render a good approximation of laboratory flows. Note however that since the temperature profile of the laminar conductive state (2c) depends on $\eta$, criterion (8) is valid only for $\eta=0.5$. In order to investigate the effect of curvature in the transition from conductive to convective heat transfer, we performed simulations as those shown in figure $4(a)$ for different values of $\eta$. We found that for all investigated curvatures $\eta \in[0.1,0.99]$ a linear relationship of the form

$$
R a_{c}(\eta, \Gamma)=a(\eta) \Gamma-b(\eta)
$$

is always satisfied, but the slope $a$ depends significantly on $\eta$ (see figure $4(b)$ ). The curve $a(\eta)$ is well approximated by the cubic function

$$
a(\eta)=-307.2 \eta^{3}+750.1 \eta^{2}-318.7 \eta+339.6
$$

The offset of the linear equation is neglected because it is small in comparison with $a(\eta) \Gamma$ for $\Gamma \geq 12.5$. 
(a)

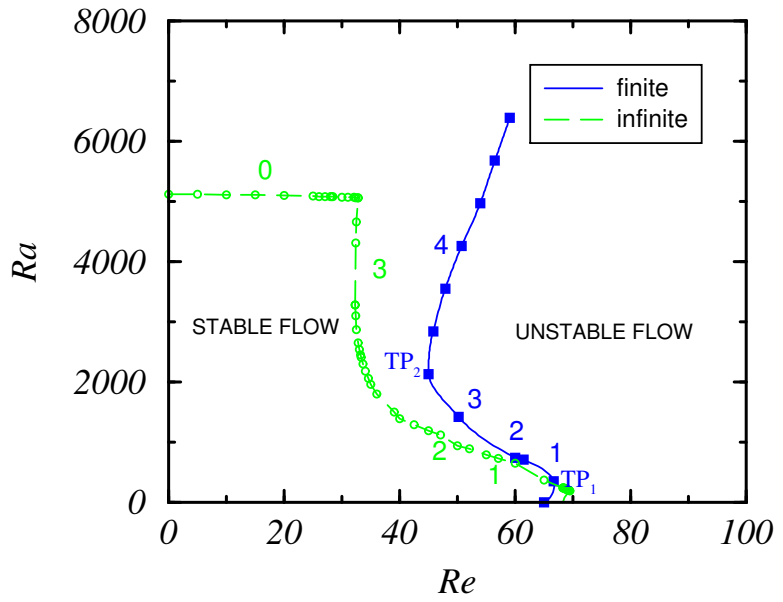

(b)

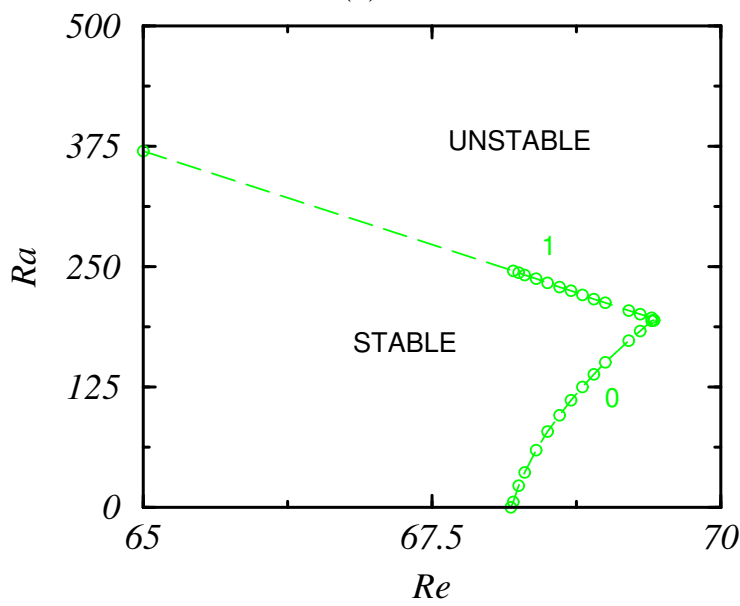

Figure 5: (color online) (a) Critical stability boundaries $\left(G_{c}\right.$ vs $R e$ ). The numbers that appear on top of the critical curves are the azimuthal modes associated to the critical perturbations. (Blue) Solid line and squares are used in the case of finite-length cylinders, whereas (green) dashed line and circles stand for the marginal stability in the case of infinite long cylinders. The working fluid is air $(\sigma=0.71)$ and the geometric parameters are $\eta=0.5$ and $\Gamma=10.0$ (in the finite case). (b) Detail of the marginal curve for low $G$ in the infinite cylinders case.

\section{Primary instability}

\subsection{Axially periodic disturbances}

The dashed line in figure 5 (a) shows the stability curve of the conductive laminar flow to axially periodic disturbances for $\eta=0.5$ and $\sigma=0.71$. Three regions with qualitatively different behaviour can be distinguished. For slow rotation the laminar flow becomes unstable to axisymmetric counter-rotating convection rolls as $R a$ increases. Buoyancy is the indirect force driving the instability, which occurs because the axial velocity profile $(2 \mathrm{~b})$ has an inflection point. At low $R a$ viscosity can stabilise the laminar flow, but as $R a$ increases the magnitude of the axial velocity grows proportionally to $R a$ until the flow becomes unstable at $R a_{c}=5120$. The instability pattern consists of axisymmetric convection rolls that travel axially in the upward direction 24. This axial drift of the rolls is caused by curvature, which makes the axial velocity profile of the conductive basic flow radially asymmetric (see Fig. 2). In the limit of vanishing curvature $\eta \rightarrow 1$ (vertical plates) the roll-pattern is steady because the system is leftright symmetric about the mid-gap [25].

As the inner-cylinder rotation speed increases the centrifugal force starts to compete with buoyancy and the laminar flow becomes first unstable to nonaxisymmetric spiral modes. Although centrifugal instabilities are typically axisymmetric (i.e. Taylor vortices), it is well known that in the presence of a sufficiently large axial velocity nonaxisymmetric spiral modes are dominant. This was first shown by Snyder [26] in TaylorCouette flow with a superimposed axial pressure gradient. In our system, as Re increases the critical Rayleigh number for the onset of these spiral modes and their wavenumber $(n)$ progressively decrease. At $(R e, R a)=(69.42,194.53)$ the critical curve for the spiral modes intersects the curve corresponding to the pure centrifugal instability (i.e. Taylor vortices with $n=0$ ). Fig. 5(b) shows a close up to the vicinity of this point.

\subsection{Axially bounded cylinders}

Following Kuo and Ball [15] we chose $\Gamma=10$, for which the transition between conductive and convective states is predicted at $R a \approx 3164$ according to $(8)$. The computed stability curve is shown as a solid line in 
figure $5(a)$ and also features an intersection point around which spiral flow and Taylor vortices compete. This is however displaced to $(R e, R a)=(66,257.02)$ because of the finite aspect-ratio. As in the periodic case and long aspect-ratio experiments of Snyder and Karlson [6], who used $\Gamma=337$, there is a region in which spiral vortices appear at lower values of $R e$ than Taylor vortices. However, this region is limited by a turning point at $(R e, R a)=(45,2272.71)$ and when $R a$ is further increased instabilities are suppressed. Here the Ekman layers cause the gradual transition to the convective basic state and enhance heat transfer without the need for instabilities. Note that this strong discrepancy with the periodic case occurs at lower $R a$ than predicted from criterion (8). However, the latter is based on the difference with the conductive temperature profile and actual profile at mid-height. We expect that to reproduce the periodic results the profile should be conductive along several $d$ in height.

\section{Heat transfer for increasing rotation speed}

The stability analysis of the previous section cannot predict the dynamics and heat transfer properties of the flow. For these purposes we performed direct numerical simulations of the Navier-Stokes equations. We first investigated $R a=1420$, so that the temperature for $\Gamma=10$ is conductive. In the periodic case we choose a cylinder length of $L_{z}=7.48$, which allows some freedom in the selection of the wavenumber of the vortex-pairs.

Figure 6 shows the sequence of emerging patterns in the periodic (upper row) and finite (lower row) cases as the Reynolds number is increased. For the periodic system the primary instability occurs at $R e \approx 39$, leading to spiral flow with azimuthal wavenumber $n=3$ (figure 6 (a)). By further increasing Re the axial flow produced by thermal buoyancy loses importance in favour of centrifugal forces and this results in secondary transitions towards spiral flow with decreasing azimuthal mode. The transition from $n=3$ to $n=2$ takes place at $R e \approx 52$, whereas the transition to $n=1$ occurs at $R e \approx 135$. The flow patterns with $n=2$ and $n=1$ are shown in figure $6(b)$ and $(c)$, respectively. For $R e \gtrsim 260$ the flow becomes quasi-periodic due to the emergence of a low frequency modulation. The resulting state preserves the spiral structure with $n=1$ and is characterised by the appearance of spatio-temporal defects (figure $6(d)$ ). Finally, a subsequent increase in $R e$ results a transition to the Taylor vortices (figure 6 (e)), which occurs for $R e \approx 280$.

The same sequence of flow states has been found in the finite case, but with slightly different Reynolds number for the transitions. The basic flow loses stability to spiral flow with $n=3$ for $R e \approx 50$ (figure 6 $(f)$ ), whereas the transitions to $n=2$ and $n=1$ occur at $R e \approx 63$ and $R e \approx 90$ respectively (figure 6 $(g)--(h)))$. The transition between spiral flow and Taylor vortices is also characterised by the presence of defects, which are much more pronounced than in the infinite cylinders case (figure $6(i)$ ). The onset of Taylor vortices (figure $6(j)$ ) takes place at $R e \approx 130$.

In figure 7 we show the heat transfer rate, normalised by the conductive rate as in equation (6), as a function of increasing Re. As the basic flow is conductive for periodic and finite boundary conditions, $N u$ is in both cases independent of $R e$ before the onset of instabilities $(R e \lesssim 50)$. In the infinite case heat transfer is purely conductive $(N u=1)$, whereas in the finite case, $N u$ is slightly higher $(N u=1.07)$ due to the heat transfer at the Ekman boundary layers. The onset of instability enhances the convective heat transfer significantly and the Nusselt number follows a power-law scaling $N u=A R e^{B}$, with $(A, B)=(0.14,0.51)$ for the finite case and $(A, B)=(0.13,0.53)$ for the periodic case.

\section{Heat transfer for increasing temperature difference}

We here investigate the dynamics and heat transfer for $R e=50$ and increasing $R a$. The upper row of figure 8 illustrates the bifurcation scenario in the periodic case. Colormaps of the temperature in longitudinal sections at $r=\frac{r_{o}+r_{i}}{2}$ are depicted. The onset of instability occurs at $R a \approx 942.88$ resulting in a spiral flow pattern with azimuthal mode number $n=2$, which is stable only in the vicinity of the critical point. A small increase in $\Delta T$ leads to the emergence of a new spiral state with $n=3$ (figure $8(a)$ ), which remains stable for $1065 \lesssim R a \lesssim 2485$. Complex spatio-temporal dynamics emerge as $R a$ is further increased $(R a>2485)$. The sequence is as follows. First, spiral states turn into wavy spiral flow patterns shown in figures $8(b)$ and 
(a)

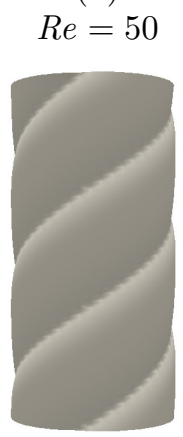

(f) (b)

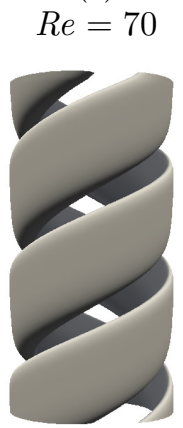

(g) (c)

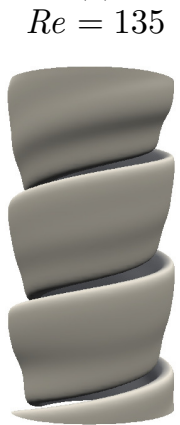

(h)

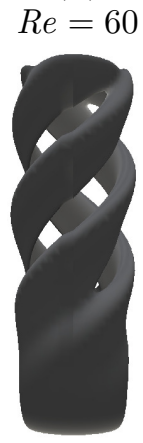

$R e=90$

(i)

(d)

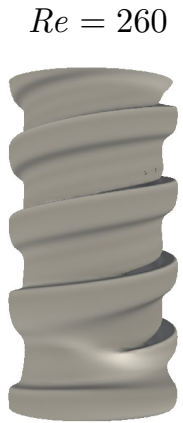

(e)

$R e=300$

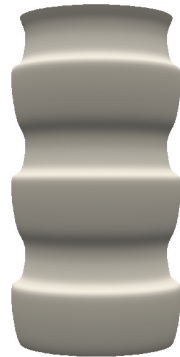

(j)
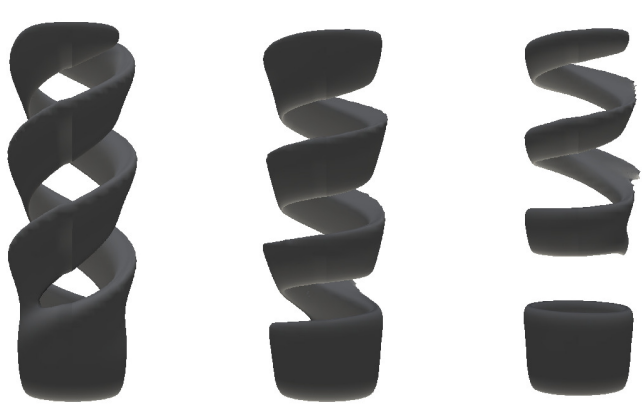

$R e=140$

Figure 6: Evolution of an isosurface of the axial velocity in the periodic (upper row) and finite (lower row) systems as $R e$ is varied and $R a=1420, \sigma=0.71, \eta=0.5, \Gamma=10$ (finite case) and $L_{z}=7.48$ (infinite case).

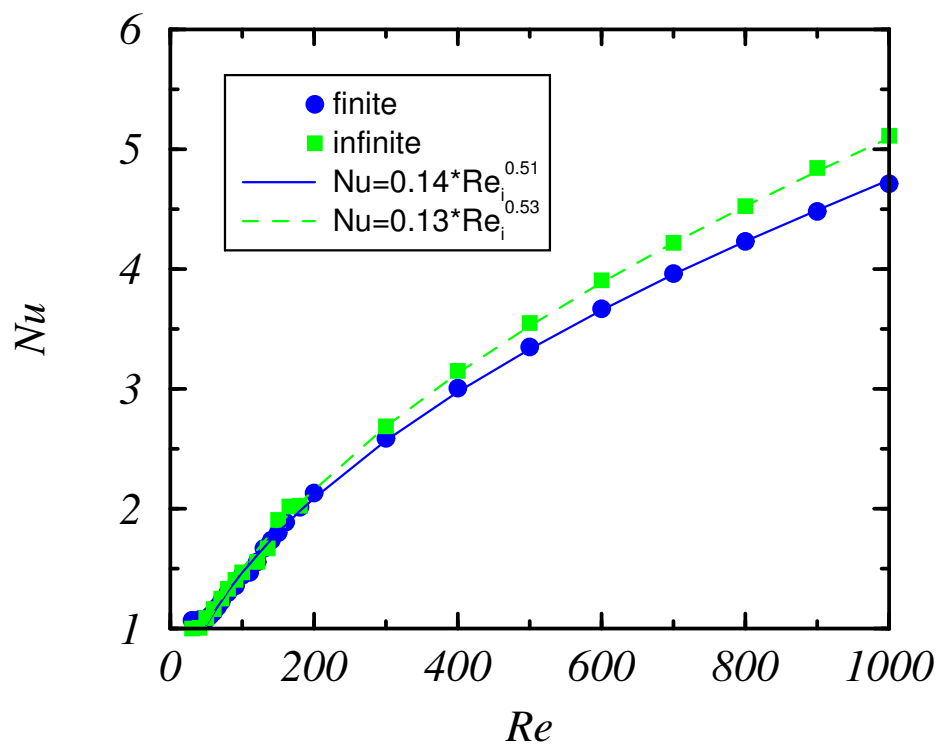

Figure 7: Variation of $N u$ with $R e$ for $R a=1420, \sigma=0.71$ and $\eta=0.5$ in the finite $(\Gamma=10)$ and infinite $\left(L_{z}=7.48\right)$ cases. 
(a)

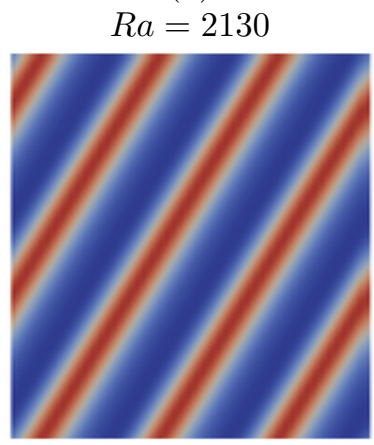

(e)

$R a=1420$

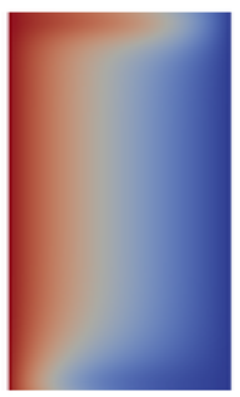

(b)

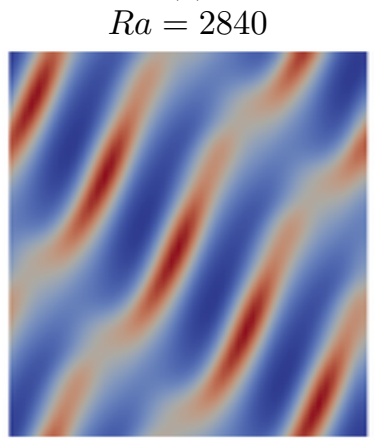

$(f)$

$R a=1775$

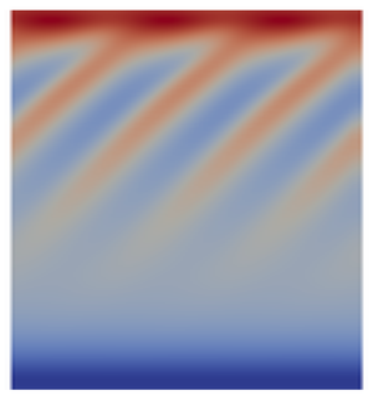

(c)

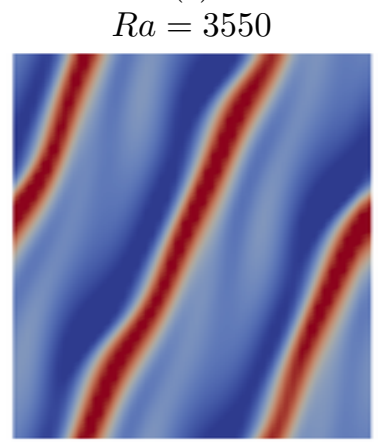

(g)

$R a=3550$

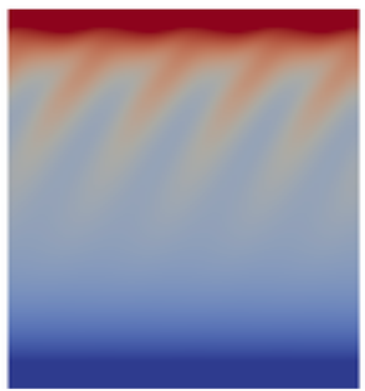

(d)

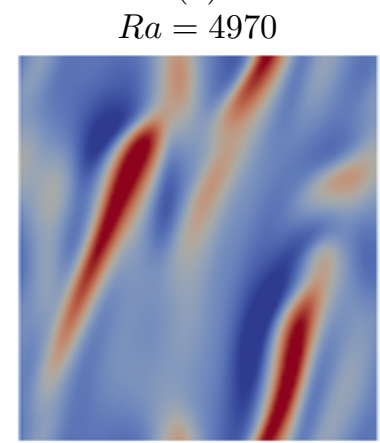

(h)

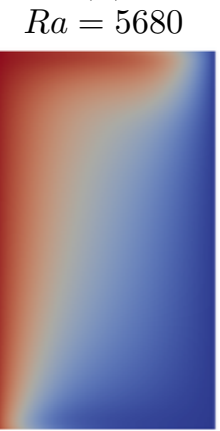

Figure 8: (color online) Evolution of the temperature in the periodic (upper row) and finite (lower row) systems as $R a$ changes and $R e=50, \sigma=0.71, \eta=0.5, \Gamma=10$ (finite case) and $L_{z}=7.48$ (infinite case). Colormaps are plotted in longitudinal sections $(\theta, z)$ at $r=\frac{r_{o}+r_{i}}{2}$, with the exception of the axisymmetric states $(e)$ and $(h)$, which are plotted in a meridional section $(r, z)$. Note that these plots have been resized and do not correspond to the aspect-ratio of the investigated system.

(c), corresponding to $R a=2840$ and $R a=3550$ respectively. Note that the dominant spiral mode changes back from $n=3$ to $n=2$ as $R a$ is increased. These flow patterns are similar to those reported in both experimental [8] and numerical studies [27] in for large $\Gamma$ and $\eta \approx 0.8$. Subsequently increasing $R a$ above the wavy spiral flow regime the flow becomes chaotic (figure $8(d)$ ).

The sequence of flow states obtained in the finite case (lower row of figure 8) is qualitatively similar to the periodic case up to the transition to the convective basic state, which is found to occur at $R a \approx 4000$, in qualitative agreement with criterion (8).

Nevertheless, there are quantitative differences. Here the conductive basic flow (figure $8(e)$ ) remains stable up to $R a \approx 1420$, where spiral flow with $n=3$ develops (figure $8(f)$ ). As the radial heating increases the dominant spiral mode changes to $n=4$ at $R a \approx 3550$ (figure $8(g)$ ). This increase in $n$ with $R a$ was previously observed and attributed to the stronger axial flow acting on the fluid because of thermal buoyancy [15. Further increasing $R a$ leads to the thinning of the thermal boundary layers at the sidewalls and the flow transitions gradually to the convective laminar flow (figure $8(h)$ ). A similar transition from spiral flow to the convective state as $\Delta T$ is increased was experimentally observed by [14] using an apparatus with $\Gamma=31.5$.

The evolution of the normalised $N u$ with $R a$ clearly illustrates the different dynamical behaviours in the finite (figure $9(a)$ ) and infinite (figure 9 (b)) systems. The largest differences occur for weak rotation $(R e=20)$. In the infinite case, $N u=1$ remains constant up to $R a \approx 7810$, indicating that heat transport is exclusively conductive up to reach the onset of instability. In contrast, in the finite case, the Ekman layers provide an efficient mechanism to transfer heat, which results in an almost linear increase of $N u$ 
(a)

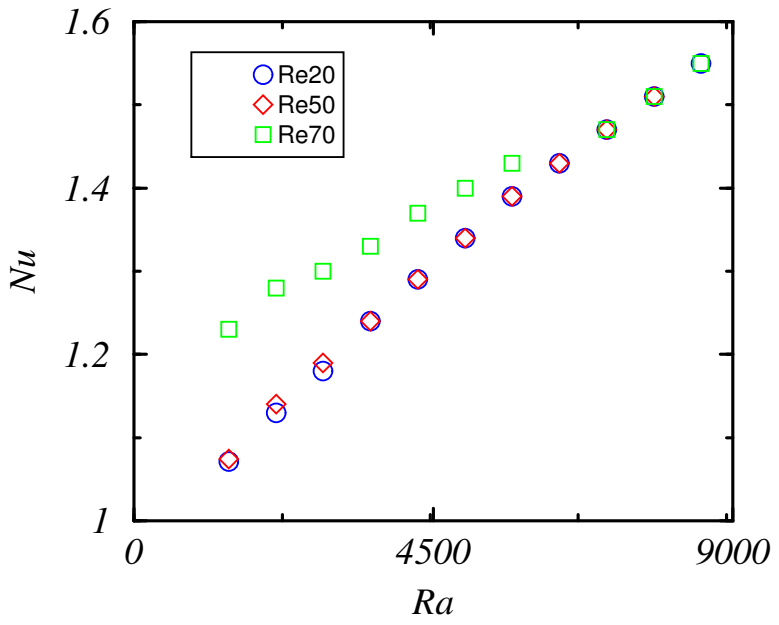

(b)

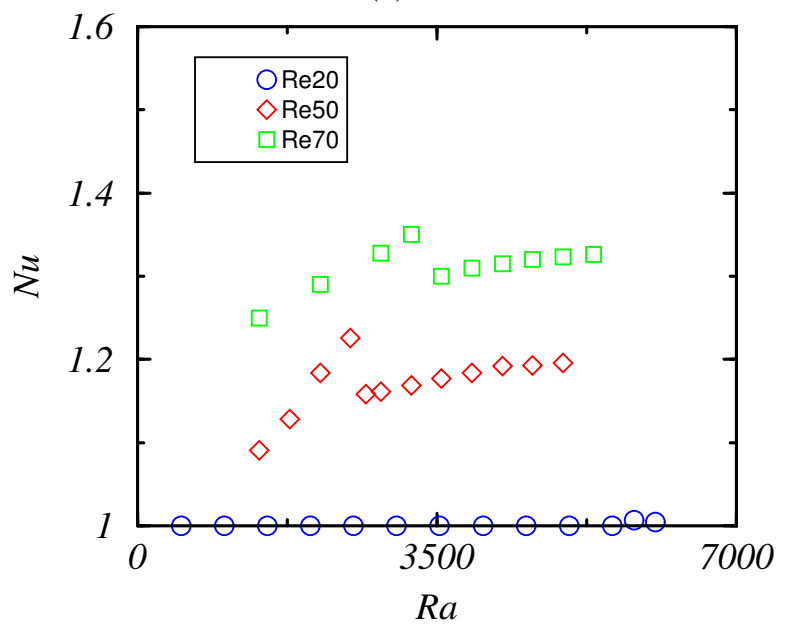

Figure 9: Variation of $N u$ with $R a$ as a function of $R e$ for $\sigma=0.71$ and $\eta=0.5$ in the $(a)$ finite $(\Gamma=10)$ and $(b)$ infinite $\left(L_{z}=7.48\right)$ systems.

with $R e$. Such growth is also observed for spiral flow, $R e=50$ and $R e=70$. When $R a$ exceeds the value corresponding to the upper part of the marginal curve in figure $5(a)$ the flow becomes again axisymmetric and $N u$ solely depends on $R a$. As a result, curves corresponding to different values of $R e$ collapse. In the infinite case, the behaviour of $N u$ within the region of spiral flow, $R e=50$ and $R e=70$, reflects the transitions described above. The spiral flow $(R a \lesssim 2750)$ resulting from the primary transition provides efficient heat transfer, leading to a rapid linear growth of $N u$ as $R a$ is increased. In this flow regime the values of $N u$ in the infinite case are slightly larger than those in the finite case. The transition towards wavy spiral flow results in a sudden decrease of the convective heat transport, consistently with the results of [11. After this initial drop, further increasing $R a$ towards fully developed turbulent flow is accompanied by a progressive growth in $N u$, which is however significantly lower than the linear growth from the spiral flow regime.

\section{Discussion}

Heat transfer in laminar flows between two concentric vertical cylinders can be of conductive or convective nature. Regardless of the curvature of the cylinders, parametrised here by the radius ratio $\eta$, the transition between these regimes is governed by the Rayleigh number $R a$ and the length-to-gap aspect ratio $\Gamma$. In particular, for a given $\Gamma$ there is a critical $R a$ above which the laminar state is convective. We here determined numerically a linear criterion (9), which applies to all geometries and is independent of the Prandtl number as long as $\Gamma \gtrsim 10$. For smaller $\Gamma$ the transition to the convective state is slightly influenced by $\sigma$ because of the stronger effect of the Ekman layers. Although the criterion was obtained for a fixed Reynolds number $R e=30$, we provided evidence that it appears valid in extended regions of the parameter regime. This was done by comparing results obtained with axially periodic boundary conditions, for which the laminar flow is always conductive, to results obtained with physical (no-slip) boundary conditions.

If the laminar flow is conductive and $R e$ is increased while $R a$ is kept fixed, our direct numerical simulations show that the transition to turbulence is very similar in the periodic and finite cases. This was shown by using $\eta=0.5, \sigma=0.71, R a=1420$ and $\Gamma=10$ in the finite case, following Kuo and Ball [15]. All their reported transitions were reproduced here, however, we found a notable difference in the onset of spiral flow, which occurs for lower $R e$ in our simulations and corresponds to a critical spiral mode $n=3$ 
instead of $n=2$. This can be explained because Kuo and Ball [15] neglected the correction of the velocity field in their simulations, so their velocity field was not mass conserving. Omitting the correction in our simulations we reproduced their results and found that its relevance diminishes as $R e$ increases, so that the secondary transitions reported here coincide with those in [15].

We further repeated these simulations by using periodic boundary conditions in a domain of length $L_{z}=7.48$. We found that the onset of instability occurs at slightly lower $R e$ than in the finite case, where the Ekman layers act as frictional layers, slowing down the internal flow and thus delaying the primary and secondary instabilities. In contrast, as the $R e$ is further increased the remaining instabilities, culminating in Taylor-vortex flow, are favoured by the presence of axial end walls. In this case the Ekman circulations at the end wall enhance the Taylor vortices, as happens in the isothermal case.

The heat transfer, quantified by the convective Nusselt number (6), follows a simple power law $(N u=$ $0.14 R e^{0.51}$ and $N u=0.13 R e^{0.53}$ in the finite and infinite cases respectively). The exponents are in good agreement with the analytical correlation provided by Dorfman [28. A similar exponent $(B=0.47)$ has also been found by Viazzo and Poncet [27] who numerically explored a region of parameter space similar to that in figure 7, but using an apparatus with $\eta=0.8$ and $\Gamma=80$. The small discrepancies between the correlations obtained in the finite and infinite cases occurs because of the Ekman layers, whose importance grows as $\Gamma$ is reduced.

If $R e$ is kept fixed while $R a$ increases, the transition to turbulence and heat transfer of the periodic and the finite system remain similar as long as criterion $(9)$ is approximately satisfied. In the finite system, further increases in $R a$ result in a transition to the laminar convective state, whereas the periodic system becomes gradually more turbulent. Thus there are two counter-acting mechanisms as $R a$ increases. Instabilities and turbulence are stimulated because of thermal driving. However, thermal driving results in a thinning of the thermal boundary layers because of end-wall effects. This quenches turbulence and causes the transition to the laminar convective state. Noteworthy, at a fixed $R a$ the laminar convective state is much more efficient in transferring heat than the turbulent flow of the periodic system.

In summary, we showed that criterion (9) is very useful in determining whether the infinite-cylinder assumption in analytical studies, or axially periodic boundary conditions in numerical studies, can be used to describe the dynamics and heat transfer of laboratory setups. This criterion applies to all geometries and fluid properties in laminar flow, and appears to provide a reasonable estimate as well in the turbulent regime. Finally, note that laminar flow in the infinite case can be modified to by adding an additional term to include stable vertical stratification [23. This should be used in simulations with periodic boundary conditions if the criterion provided here is not satisfied.

\section{Conclusions}

The role of axial boundaries in laterally heated and rotating flows was investigated with linear stability analyses and direct numerical simulations. Both periodic and no-slip boundary condi- tions were considered in the axial direction. The main results can be summarised as follows:

- Criterion (9) determines whether laminar heat transfer in an axially bounded system is of conductive or convective nature. It is independent of Prandtl number and robust with Reynolds number as long as $\Gamma \gtrsim 15$.

- If the laminar state is conductive axially periodic boundary con- ditions correctly describe the dynamics and heat transfer in lab- oratory setups. If the laminar state is convective the infinite-cylinder approximation may be used but the basic flow must be modified to include axial stratification [23].

- A variety of flow patterns was observed. The underlying flow instabilities are driven by the interaction of two physical mech- anisms: thermal buoyancy generates an inflectional axial veloc- ity profile, whereas the inner-cylinder rotation promotes centrifugal instability.

\section{Conflict of interest}

None declared. 


\section{Acknowledgments}

This work was supported by the Spanish Government grant FIS2013-40880-P and BES-2010-041542. Part of this work was done during the visit of J.M. Lopez to the Institute of Science and Research (IST) in Klosterneuburg (Austria) whose kind hospitality is warmly appreciated. We thank Red Espaola de Supercomputacin (RES) for the computational resources provided.

\section{References}

[1] M. Lappa, Rotating Thermal Flows in Natural and Industrial Processes, John Wiley and Sons, 2012.

[2] F. Kreith, Convection heat transfer in rotating systems, Advances in Heat Transfer 5 (1968) 129-251.

[3] P. Singer, Techniques of low pressure chemical vapor deposition, Semiconductor, Int. (1984) $72-77$.

[4] C. Vivès, Effects of a forced Couette flow during the controlled solidification of a pure metal, International Journal of Heat and Mass Transfer 31 (10) (1988) 2047-2062.

[5] F. Selimefendigil, H. F.O., Forced convection of ferrofluids in a vented cavity with a rotating cylinder, International Journal of Thermal Sciences 86 (0) (2014) 258-275.

[6] Snyder, H. A. and Karlsson, S. K. F, Experiments on the stability of Couette motion with a radial thermal gradient, Phys. Fluids 7 (1964) 1696.

[7] Sorour, M. M. and Coney, J. E. R., The effect of temperature gradient on the stability of flow between vertical concentric rotating cylinders, J. Mech. Eng. Sci. 21 (1979) 403-409.

[8] Lepiller, V. and Goharzadeh, A. and Prigent, A. and Mutabazi, I., Weak temperature gradient effect on the stability of the circular Couette flow, The European Physical Journal B 61 (2008) 445-455.

[9] M. E. Ali, P. D. Weidman, On the stability of circular Couette-flow with radial heating, J. Fluid Mech. 220 (1990) 53-84.

[10] H. N. Yoshikawa, M. Nagata, I. Mutabazi, Instability of the vertical annular flow with a radial heating and rotating inner cylinder, Phys. Fluids 25 (2013) 114104.

[11] Kedia, R. and Hunt, M. L. and Colonius, T., Numerical Simulations of Heat Transfer in Taylor-Couette Flow, J. Heat Transfer 120 (1998) 65-71.

[12] K. S. Ball, B. Farouk, On the development of Taylor vortices in a vertical annulus with a heated rotating inner cylinder, Int. J. Numer. Meth. Fluids 7 (1987) 857-867.

[13] K. S. Ball, B. Farouk, Bifurcation phenomena in Taylor-Couette flow with buoyancy effects, J. Fluid Mech. 197 (1988) 479-501.

[14] K. S. Ball, B. Farouk, A flow visualization study of the effects of buoyancy on Taylor vortices, Phys. Fluids A 1 (1989) $1502-1507$.

[15] Kuo, D.-C. and Ball, K. S., Taylor-Couette flow with buoyancy: Onset of spiral flow, Phys. Fluids 9 (1997) $2872-2884$.

[16] J. M. Lopez, F. Marques, M. Avila, The Boussinesq approximation in rapidly rotating fluids, J. Fluid Mech. 737 (2013) $56-77$.

[17] Shi, L. and Rampp, M. and Hof, B. and Avila, M., A Hybrid MPI-OpenMP Parallel Implementation for pseudospectral simulations with application to Taylor-Couette Flow, Computers and Fluids 106 (2015) 1-11.

[18] Avila, M. and Grimes, M. and Lopez, J. M. and Marques, F., Global endwall effects on centrifugally stable flows, Phys. Fluids 20 (2008) 104104.

[19] J. M. Lopez, Numerical modeling of simple laboratory experiments of rotating flow, Ph.D. thesis, Applied Physics, UPC, Barcelona (February 2015).

[20] H. P. Greenspan, The Theory of Rotating Fluids, 2nd Edition, Cambridge University Press, 1968.

[21] Hide, R. and Mason, P. J., Sloping convection in a rotating fluid, Advances in Physics 24 (1975) 47-100.

[22] R. W. Thomas, G. de Vahl Davis, Natural convection in annular and rectangular cavities: a numerical study, Proc. 4th Intl Heat Transfer Conf., Paris, 1970.

[23] M. E. Ali, G. B. McFadden, Linear stability of cylindrical Couette flow in the convection regime, Phys. Fluids 17 (5) (2005) 054112.

[24] de Vahl Davis, G. and Thomas, R. W., Natural convection between concentric vertical cylinders, Phys. Fluids Suppl. II (1969) 198-207.

[25] J. Elder, Laminar free convection in a vertical slot, J. Fluid Mech. 23 (01) (1965) 77-98.

[26] H. A. Snyder, Experiments on the stability of spiral flow at low axial reynolds numbers, Proc. Roy. Soc. Lond. A 265 (1321) (1962) 198-214.

[27] S. Viazzo, S. Poncet, Numerical simulation of the flow stability in a high aspect ratio Taylor-Couette system submitted to a radial temperature gradient, Computers \& Fluids 101 (2014) 15-26.

[28] Dorfman, L.A., Hydrodynamic Resistance and the Heat Loss of Rotating Solids, Oliver \& Boyd, 1963. 